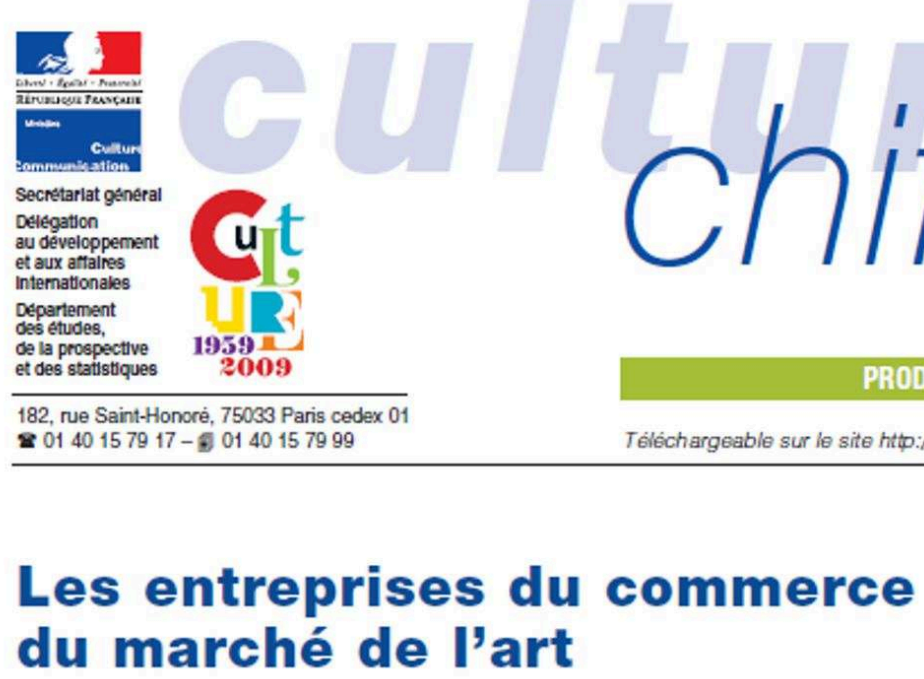

François Rouet ${ }^{*}$

Les principales activités commerciales du marché de l'art ne sont pas identifiées en tant que telles dans la nomenclature d'activités française en vigueur $^{1}$, ce qui empêche toute description statistique des activités et des entreprises qui les exercent (galeristes, antiquaires, libraires d'anciens...) à partir des enquêtes sectorielles annuelles, portant sur le commerce, réalisées par l'Insee.

Il est cependant possible d'obtenir, à partir de ces enquêtes, une série d'indications statistiques, jusqu'ici inédites, sur les activités et les entreprises concernées, grâce à une exploitation spécifique menée par le département Commerce de l'Insee à la demande du DEPS. Ainsi, l'activité commerciale d'environ 15000 entreprises de commerce d'art qui réalisent un chiffre d'affaires de 1,9 milliard d'euros grâce à cette activité a pu être étudiée. Les résultats portent essentiellement sur quatre activités commerciales: le commerce d'œuvres d'art contemporain, la vente d'antiquités, d'objets d'art et de meubles anciens, la vente d'objets de récupération et enfin la vente de livres anciens et d'occasion, activités relevant de trois codes Naf différents (52.4Z, $52.5 \mathrm{Z}$ et $52.6 \mathrm{E})$. On dispose également de données sur deux activités commerciales très spécifiques : la vente de pièces de collection et celle de timbres-poste de collection ${ }^{2}$.

\begin{tabular}{|c|}
\hline $\begin{array}{l}\text { Vers une appréciation } \\
\text { plus assurée du marché de l'art }\end{array}$ \\
\hline 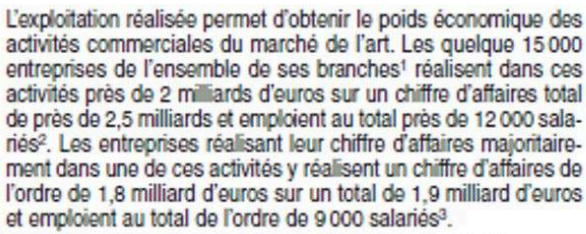 \\
\hline 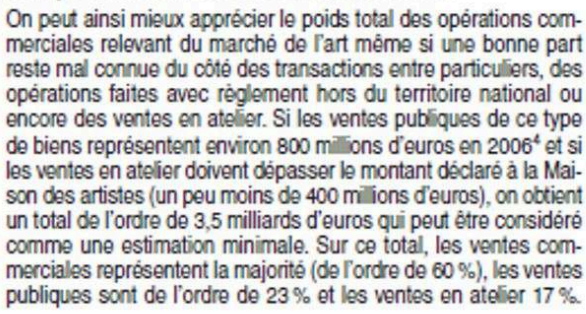 \\
\hline 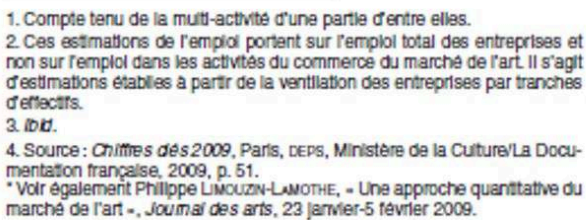 \\
\hline
\end{tabular}

\footnotetext{
* Chargé d'étude au pars.

1. La présente exploitation ayant êté réalisée sur la base de données relatives à l' année 2006, la nomenclature en vigueur était la Naf 2003, et non pas la Naf 2008 rév. 2, en vigueur depuis le le janvier 2008.

2. Les sociétés de vente volontaire aux enchères publiques (code $74.8 \mathrm{~K}$ ) qui sont teaues de fournir des informations régulières sur leur activité au Conseil des ventes, organisme de régulation, ne sont en revanche pas intégrées à l'analyse.
} 


\section{Les entreprises du commerce du marché de l'art}

\section{François Rouet}

Éditeur : Département des études, de la prospective et des statistiques

Lieu d'édition : Paris

Année d'édition : 2009

Date de mise en ligne : 21 septembre 2015

Collection : Culture chiffres

ISBN électronique : 9782111398450

\section{Sbooks}

http://books.openedition.org

\section{Édition imprimée}

Date de publication : 1 avril 2009

Nombre de pages : 8

\section{Référence électronique}

ROUET, François. Les entreprises du commerce du marché de l'art. Nouvelle édition [en ligne]. Paris : Département des études, de la prospective et des statistiques, 2009 (généré le 25 avril 2021).

Disponible sur Internet : <http://books.openedition.org/deps/580>. ISBN : 9782111398450 


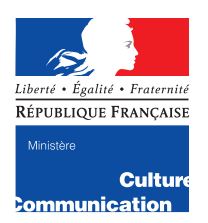

Secrétariat général Délégation au développement et aux affaires internationales Département des études, de la prospective et des statistiques
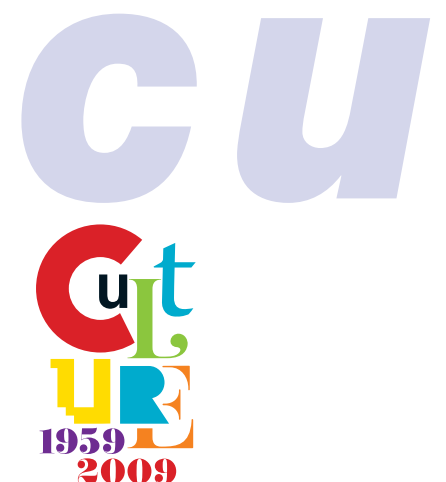

2009

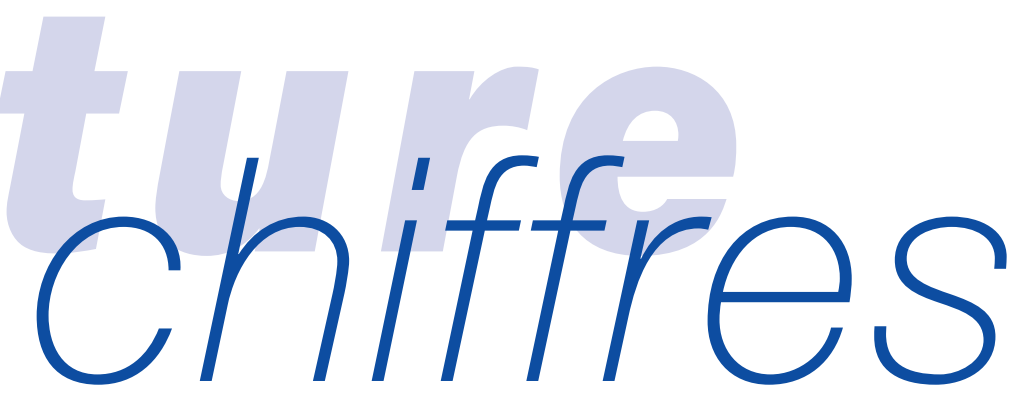

PRODUCTION, DIFFUSION ET MARCHÉS

\section{Les entreprises du commerce du marché de l'art}

\section{François Rouet*}

Les principales activités commerciales du marché de l'art ne sont pas identifiées en tant que telles dans la nomenclature d'activités française en vigueur $^{1}$, ce qui empêche toute description statistique des activités et des entreprises qui les exercent (galeristes, antiquaires, libraires d'anciens...) à partir des enquêtes sectorielles annuelles, portant sur le commerce, réalisées par l'Insee.

Il est cependant possible d'obtenir, à partir de ces enquêtes, une série d'indications statistiques, jusqu'ici inédites, sur les activités et les entreprises concernées, grâce à une exploitation spécifique menée par le département Commerce de l'Insee à la demande du DEPS. Ainsi, l'activité commerciale d'environ 15000 entreprises de commerce d'art qui réalisent un chiffre d'affaires de 1,9 milliard d'euros grâce à cette activité a pu être étudiée. Les résultats portent essentiellement sur quatre activités commerciales: le commerce d'œuvres d'art contemporain, la vente d'antiquités, d'objets d'art et de meubles anciens, la vente d'objets de récupération et enfin la vente de livres anciens et d'occasion, activités relevant de trois codes Naf différents (52.4Z, $52.5 \mathrm{Z}$ et $52.6 \mathrm{E})$. On dispose également de données sur deux activités commerciales très spécifiques : la vente de pièces de collection et celle de timbres-poste de collection ${ }^{2}$.

\section{Vers une appréciation plus assurée du marché de l'art*}

L'exploitation réalisée permet d'obtenir le poids économique des activités commerciales du marché de l'art. Les quelque 15000 entreprises de l'ensemble de ses branches ${ }^{1}$ réalisent dans ces activités près de 2 milliards d'euros sur un chiffre d'affaires total de près de 2,5 milliards et emploient au total près de 12000 salarié $^{2}$. Les entreprises réalisant leur chiffre d'affaires majoritairement dans une de ces activités y réalisent un chiffre d'affaires de l'ordre de 1,8 milliard d'euros sur un total de 1,9 milliard d'euros et emploient au total de l'ordre de 9000 salariés $^{3}$.

On peut ainsi mieux apprécier le poids total des opérations commerciales relevant du marché de l'art même si une bonne part reste mal connue du côté des transactions entre particuliers, des opérations faites avec règlement hors du territoire national ou encore des ventes en atelier. Si les ventes publiques de ce type de biens représentent environ 800 millions d'euros en $2006^{4}$ et si les ventes en atelier doivent dépasser le montant déclaré à la Maison des artistes (un peu moins de 400 millions d'euros), on obtient un total de l'ordre de 3,5 milliards d'euros qui peut être considéré comme une estimation minimale. Sur ce total, les ventes commerciales représentent la majorité (de l'ordre de $60 \%$ ), les ventes publiques sont de l'ordre de $23 \%$ et les ventes en atelier $17 \%$.

1. Compte tenu de la multi-activité d'une partie d'entre elles.

2. Ces estimations de l'emploi portent sur l'emploi total des entreprises et non sur l'emploi dans les activités du commerce du marché de l'art. II s'agit d'estimations établies à partir de la ventilation des entreprises par tranches d'effectifs.

3. Ibid.

4. Source: Chiffres clés 2009, Paris, DEPS, Ministère de la Culture/La Documentation française, 2009, p. 51.

* Voir également Philippe LimouzIN-LAMOTHE, « Une approche quantitative du marché de l'art », Journal des arts, 23 janvier-5 février 2009.

\footnotetext{
* Chargé d'étude au DEPS.

1. La présente exploitation ayant été réalisée sur la base de données relatives à l'année 2006, la nomenclature en vigueur était la Naf 2003, et non pas la Naf 2008 rév. 2, en vigueur depuis le $1^{\text {er }}$ janvier 2008.

2. Les sociétés de vente volontaire aux enchères publiques (code $74.8 \mathrm{~K}$ ) qui sont tenues de fournir des informations régulières sur leur activité au Conseil des ventes, organisme de régulation, ne sont en revanche pas intégrées à l'analyse.
} 


\section{UNE POPULATION IMPORTANTE DE COMMERCES SPÉCIALISÉS}

Les résultats permettent d'apprécier l'ampleur de la population des commerces réalisant majoritairement leur activité dans l'une de ces activités : ce sont près de 13500 entreprises qui exercent majoritairement leur activité dans l'une de ces activités. La population la plus importante est celle qui pratique majoritairement la vente d'antiquités (70\% du total) suivie, de très loin, par les entreprises qui se consacrent à la vente d'objets de récupération $(12 \%)$ et par les galeries d'art contemporain $(9 \%)$ (graphique 1).

Le taux de spécialisation de ces commerces est fort et dépasse en moyenne $95 \%$ de l'activité dans la plupart des catégories, à l'exception de la brocante en magasin et des vendeurs de pièces et de timbres de collection. Cette forte spécialisation s'applique à des chiffres d'affaires moyens très variables d'une catégorie à l'autre mais particulièrement élevés pour les galeries d'art contemporain et les commerces d'antiquités en magasin (respectivement $315000 €$ et $207000 €$ contre $144000 €$ pour l'ensemble) (tableau 1).

Enfin, dans l'ensemble des activités à l'exception de la vente d'antiquités, les entreprises spécialisées (c'est-à-dire réalisant la majorité de leur offre d'affaires dans l'activité) sont généralement d'une taille inférieure à celle des entreprises qui exercent les mêmes activités de manière minoritaire. C'est particulièrement le cas pour la vente d'œuvres d'art contemporain, de pièces de collection et, surtout, de livres anciens ou d'occasion (voir annexe 2).

Il est probable que les entreprises exerçant majoritairement leur activité dans l'ensemble de ces acti-

\section{Graphique 1 - Répartition des entreprises exerçant majoritairement une activité de commerce de l'art selon les postes de la nomenclature Naf}

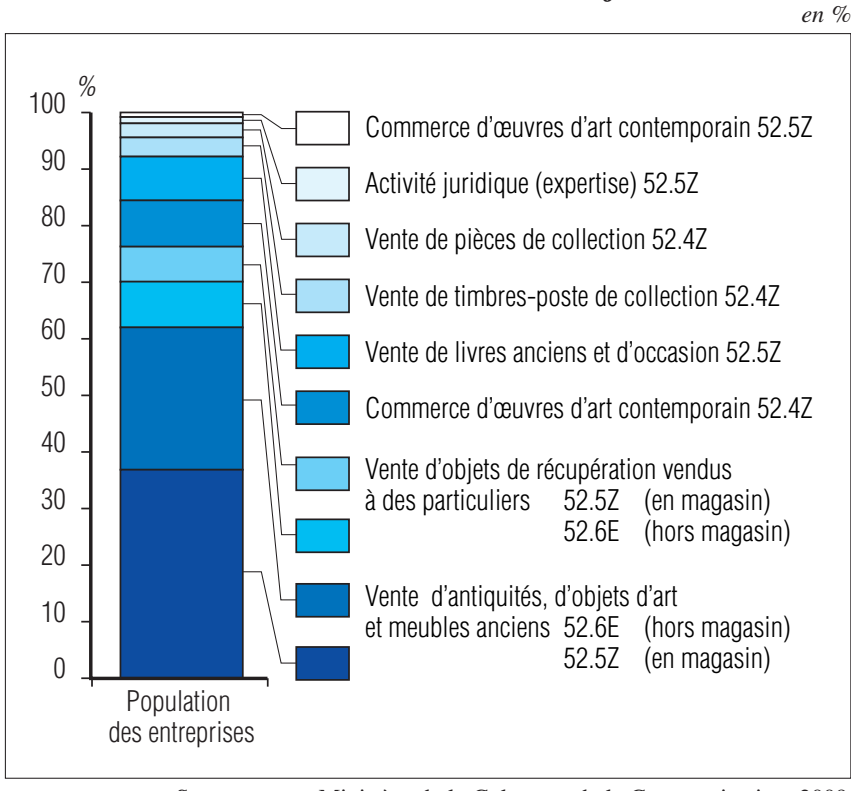

Source : DEPS, Ministère de la Culture et de la Communication, 2009

vités prises globalement sont encore plus nombreuses car l'exercice concomitant de plusieurs de ces activités semble fréquent (antiquités et livres anciens ou pièces de collection, brocante et livres d'occasion...). La population supplémentaire à prendre alors en compte, bien inférieure à celle des entreprises ayant une activité minoritaire dans l'une au moins des activités répertoriées soit un peu plus de 3000 (3 104), pourrait être de l'ordre de quelques centaines.

\section{Tableau 1 - Les entreprises exerçant une activité du commerce de l'art comme activité majoritaire}

\begin{tabular}{|c|c|c|c|}
\hline Activité & $\begin{array}{c}\text { Nombre } \\
\text { d'entreprises }\end{array}$ & $\begin{array}{l}\text { Chiffre d'affaires } \\
\text { total moyen } \\
\text { (en milliers d'euros) }\end{array}$ & $\begin{array}{c}\text { Taux de spécialisation } \\
\text { dans l'activité } \\
\text { (en \%) }\end{array}$ \\
\hline Vente d'œuvres d'art contemporain ${ }^{*}$ & 1170 & 315 & 99 \\
\hline $\begin{array}{l}\text { Vente d'antiquités, objets d'art et meubles anciens } \\
\text { - dont en magasin } \\
\text { - dont hors magasin }\end{array}$ & $\begin{array}{l}9425 \\
5696 \\
3729\end{array}$ & $\begin{array}{r}142 \\
207 \\
44\end{array}$ & $\begin{array}{l}95 \\
95 \\
98\end{array}$ \\
\hline $\begin{array}{l}\text { Vente d'objets de récupération } \\
\text { - dont en magasin } \\
\text { - dont hors magasin }\end{array}$ & $\begin{array}{r}1630 \\
591 \\
1039\end{array}$ & $\begin{array}{r}74 \\
125 \\
29\end{array}$ & $\begin{array}{r}76 \\
73 \\
100\end{array}$ \\
\hline Vente de livres anciens et d'occasion & 802 & 80 & 95 \\
\hline Vente de pièces de collection & 266 & 139 & 78 \\
\hline Vente de timbres-poste de collection & 212 & 113 & 79 \\
\hline Total & 13505 & 144 & 95 \\
\hline
\end{tabular}

Source : DEPS, Ministère de la Culture et de la Communication, 2009 


\section{DES COMMERCES SPÉCIALISÉS \\ QUI RÉALISENT L'ESSENTIEL DE CES ACTIVITÉS}

Les activités commerciales étudiées sont très largement le fait des entreprises qui les exercent de manière majoritaire. D'abord, la plus grande partie des entreprises concernées par au moins une activité (appartenant à la branche) réalisent la majorité de leur chiffre d'affaires dans cette activité : 13505 sur 16609 soit $81 \%$ (voir tableau 2, ligne 1). Cette caractéristique concerne particulièrement le commerce d'antiquités et d'œuvres d'art contemporain ; seule la vente de timbres de collection y déroge (39\%).
Le même phénomène s'observe concernant le chiffre d'affaires dans chaque activité : il est réalisé en quasi-totalité par des entreprises qui en font leur activité majoritaire : $97 \%$ pour l'ensemble, $79 \%$ au minimum pour la vente de timbres-poste de collection (tableau 2, ligne 2, graphiques 2 et 3). Il en va de même, quoique dans une moindre mesure, pour les effectifs salariés totaux des entreprises, avec un taux global de $76 \%$.

Les entreprises exerçant majoritairement leur activité dans l'une de ces activités sont très spécialisées (95\% en moyenne toutes activités confondues). Il en va de même pour l'ensemble des entreprises concernées par une activité : le taux de spécialisation est de $79 \%$ toutes activités confondues,

Tableau 2-Les principales activités du marché de l'art

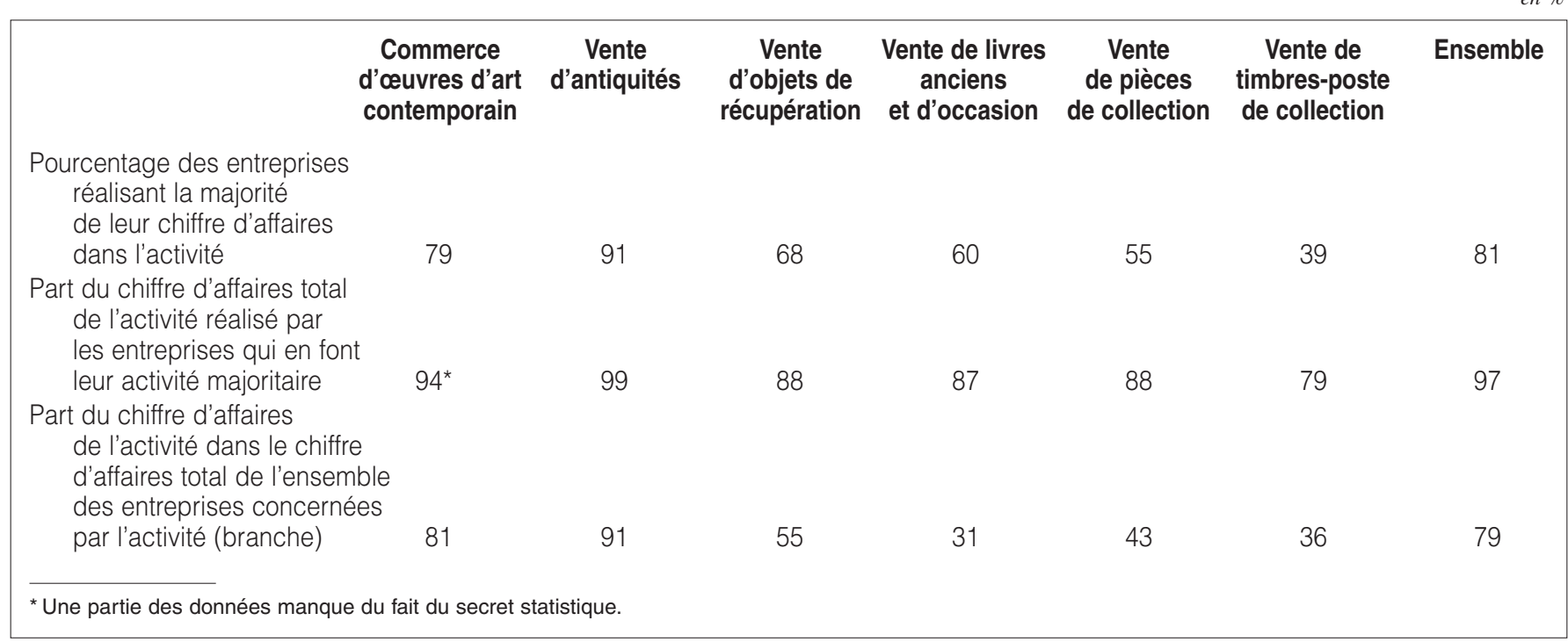

Source : DEPS, Ministère de la Culture et de la Communication, 2009

Graphique 2 - Part du chiffre d'affaires réalisé par les entreprises avec le commerce de l'art

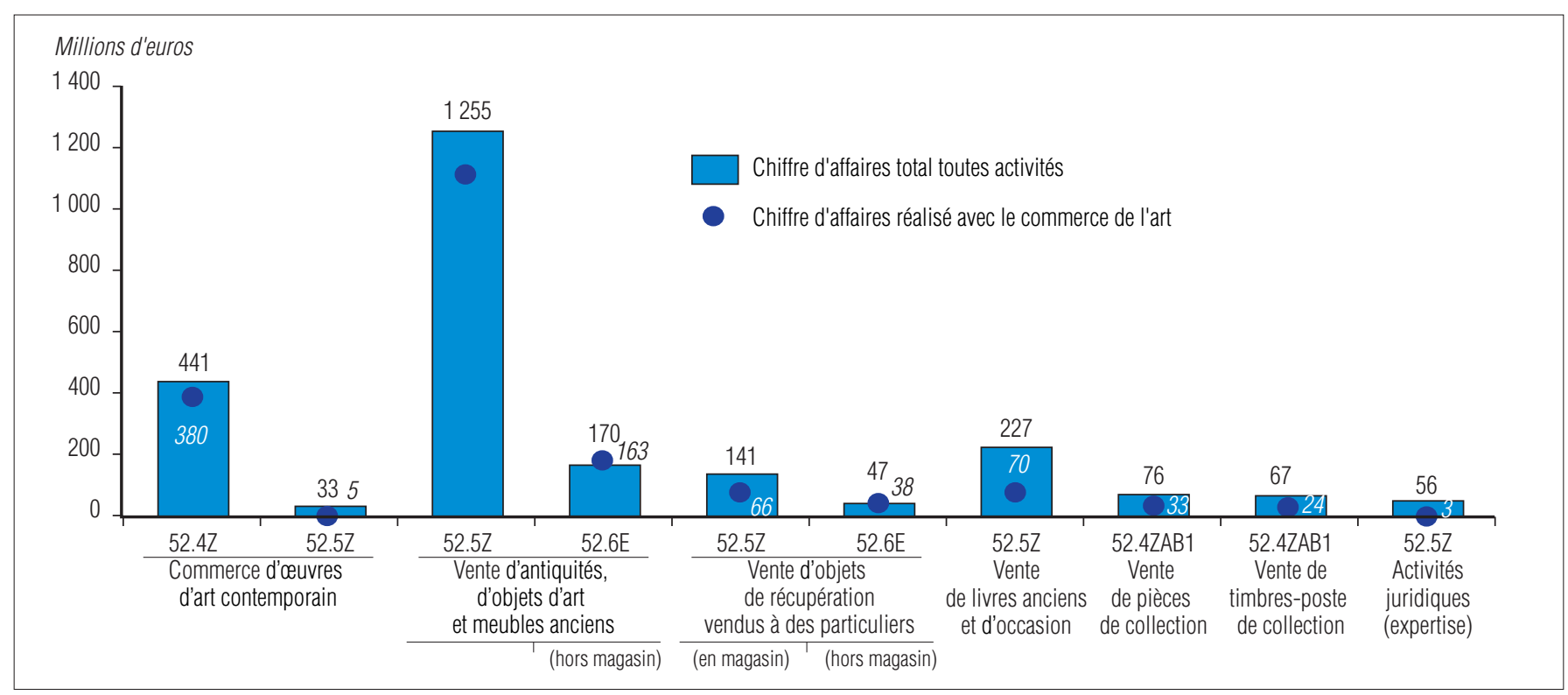

Source : DEPS, Ministère de la Culture et de la Communication, 2009 
Graphique 3 - Poids des entreprises spécialisées dans l'ensemble des entreprises de la branche et dans le chiffre d'affaires de la branche

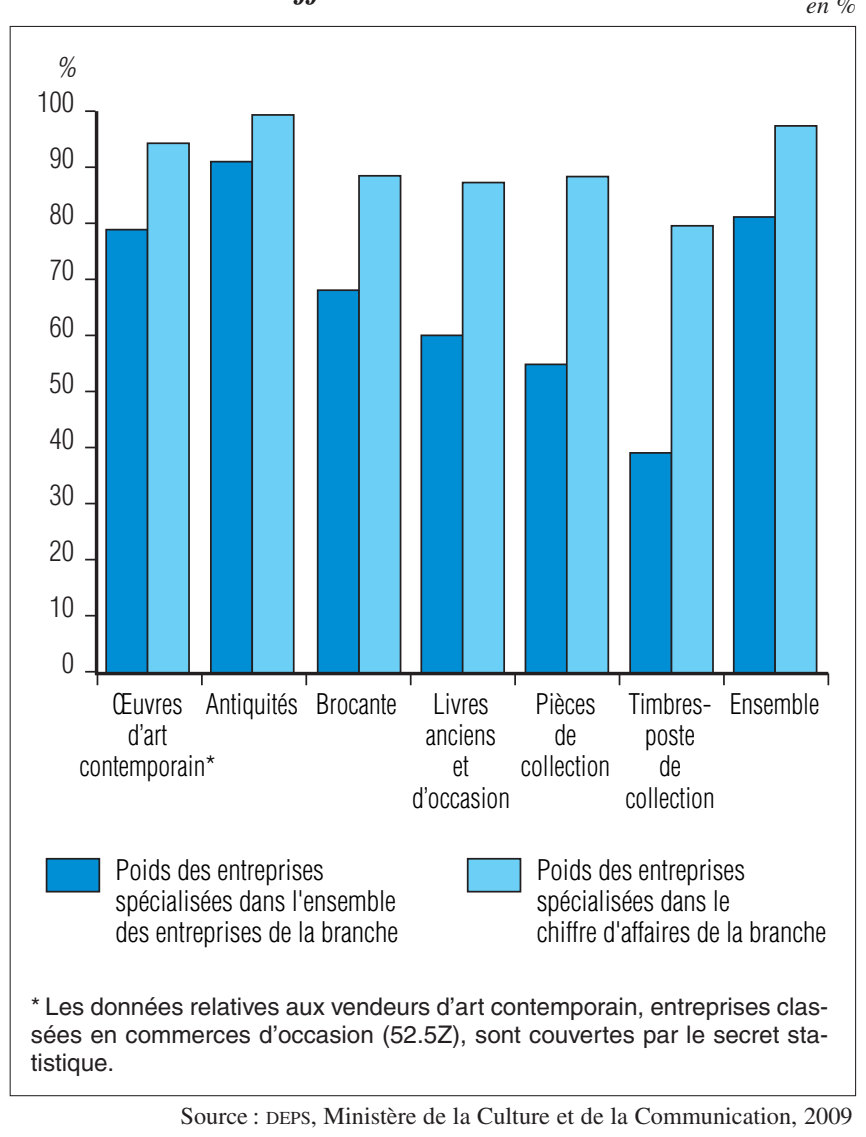

il est tout particulièrement élevé pour le commerce d'œuvres d'art contemporain $(81 \%)$ et la vente d'antiquités (91\%) (tableau 2, ligne 3). Il l'est nettement moins pour les autres activités, en particulier pour la vente de livres anciens ou d'occasion (31\%).

\section{ApProche PAR ACTIVITÉ}

\section{Le commerce d'œuvres d'art}

contemporain : 1491 entreprises réalisent un chiffre d'affaires de $\mathbf{3 8 5}$ millions d'euros

Deux types d'entreprises, relevant de deux activités distinctes de la Naf (52.4Z et 52.5Z), ont des activités de vente d'œuvres d'art contemporain. La différence entre ces deux sous-populations est très nette, au regard de leur importance numérique, de leur place dans la vente d'œuvres d'art contemporain et de leur degré de spécialisation dans cette activité.

Que l'on retienne la population totale (1 491 entreprises et 385 millions d'euros) ou la seule population des entreprises totalement spécialisées dans l'art contemporain (1170 entreprises et 363 millions d'euros), on obtient un ordre de grandeur très proche de celui des diffuseurs cotisant à la Maison des artistes du fait de leurs ventes d'art contemporain (1237 entreprises et 345 millions d'euros en 2005).

\section{Entreprises relevant du commerce divers (52.4Z)}

1356 entreprises réalisent 380 millions d'euros de chiffre d'affaires dans cette activité, pour un chiffre d'affaires total de 441 millions d'euros. Le chiffre d'affaires moyen dans cette activité est de $280000 €$ soit $86 \%$ du chiffre d'affaires moyen total. Moins de $10 \%$ de ces entreprises emploient plus de 5 salariés.

$\mathrm{Au}$ sein de cette première sous-population, la distinction suivant la part du chiffre d'affaires consacrée au commerce d'art commun est significative : parmi ces entreprises, 1170 entreprises (soit $86 \%$ d'entre elles) réalisent au moins $50 \%$ de leur chiffre d'affaires avec la vente d'œuvres d'art contemporain avec un chiffre d'affaires moyen de $310000 €$ sur $315000 €$, ce qui témoigne d'une spécialisation quasi complète (98\%). Par ailleurs, on compte parmi elles 81 entreprises employant plus de 5 salariés mais 785 n'en employant aucun.

À l'opposé, 186 entreprises réalisent moins de $50 \%$ de leur chiffre d'affaires en vente d'art contemporain, ce qui représente un chiffre d'affaires moyen de $93000 €$ pour un chiffre d'affaires total moyen de $398000 €$, sensiblement plus élevé que pour les précédentes. La part du chiffre d'affaires réalisée dans le commerce d'œuvres d'art contemporain est de $23 \%$ du chiffre d'affaires total de ces entreprises, soit un montant total de 17 millions d'euros. 
Entreprises relevant du commerce

\section{de détail de biens d'occasion (52.5Z)}

135 entreprises réalisent 5 millions d'euros seulement de chiffre d'affaires en vente d'œuvres d'art contemporain. Ces entreprises réalisent en moyenne $37000 €$ de leur chiffre d'affaires en vente d'œuvres d'art contemporain, sur un chiffre d'affaires total moyen de $244000 €$, soit seulement $15 \%$. Seulement $4 \%$ de ces entreprises emploient plus de 5 salariés tandis que $56 \%$ n'en emploient aucun. Le nombre d'entreprises réalisant plus de $50 \%$ de leur chiffre d'affaires en vente d'œuvres d'art contemporain est très réduit.

\section{La vente d'antiquités, d'objets d'art} et meubles anciens : 10394 entreprises réalisent un chiffre d'affaires

\section{de 1,281 milliard d'euros}

Les entreprises concernées relèvent de deux positions différentes au sein de la Naf. Ces deux sous-populations d'entreprises numériquement importantes présentent les caractéristiques communes d'être très spécialisées dans la vente d'antiquités, d'œuvres d'art et de meubles anciens et d'employer peu de personnel. En revanche, elles diffèrent très sensiblement quant au chiffre d'affaires moyen réalisé dans cette activité et, partant, quant à leur contribution respective au chiffre d'affaires de la branche.

\section{Entreprises relevant de la vente au détail de biens d'occasion (52.5Z)}

6203 entreprises pèsent 1,135 milliard d'euros dans cette activité sur un chiffre d'affaires total de 1,255 milliard d'euros. Le chiffre d'affaires moyen réalisé par la vente d'antiquités est ainsi de $183000 €$ sur un total de $202000 €$, ce qui témoigne d'une forte spécialisation pour $91 \%$ des entreprises. Parmi celles-ci, $3 \%$ seulement emploient plus de 5 salariés et $71 \%$ n'ont pas recours à l'emploi salarié.

La quasi-totalité de ces entreprises (92\%) réalisent plus de $50 \%$ de leur chiffre d'affaires avec la vente d'antiquités, d'objets d'art et de meubles anciens. Le chiffre d'affaires moyen de la vente d'antiquités est de $196000 €$, soit $95 \%$ du chiffre d'affaires global moyen (207 $000 €)$, ce qui signale une très forte spécialisation. Parmi ces entreprises, 152 emploient plus de 5 salariés tandis que 4097 n'en emploient aucun.
Les 507 autres entreprises réalisent moins de $50 \%$ de leur chiffre d'affaires de la vente d'antiquités, en moyenne $35000 €$ sur $152000 €$ soit $23 \%$ : il s'agit d'entreprises au chiffre d'affaires sensiblement plus faible. Le chiffre d'affaires total réalisé en antiquités, objets d'art et meubles anciens s'avère également très faible : 17 millions d'euros.

\section{Entreprises relevant de la vente au détail hors magasin (52.6E)}

4191 entreprises réalisent un chiffre d'affaires dans l'activité de 163 millions d'euros seulement. Leur chiffre d'affaires moyen réalisé dans la vente d'antiquités est de $39000 €$ (pour un chiffre d'affaires total moyen de $41000 €$ ), soit $96 \%$ de l'ensemble de leur activité, ce qui atteste, là aussi, d'une très forte spécialisation, mais pour un niveau d'affaires beaucoup plus faible. Il faut souligner qu'aucune de ces entreprises n'emploie de salarié.

La quasi-totalité $(89 \%)$ de ces entreprises (3 729) réalise plus de $50 \%$ du chiffre d'affaires dans cette activité, soit en moyenne $43000 €$ sur $44000 €(98 \%)$. Les 462 autres entreprises n'en font pas leur activité principale et n'en tirent en moyenne qu'un chiffre d'affaires de $3000 €$ sur $11000 €$, soit $20 \%$.

Enfin, 779 entreprises réalisent des commissions sur vente de ces mêmes biens d'antiquités, et réalisent un faible chiffre d'affaires de 33 millions d'euros. Le chiffre d'affaires moyen pour cette activité $(42000 €)$ ne représente que $7 \%$ de leur chiffre d'affaires total $(243000 €)$. Au-delà des 104 entreprises qui réalisent la moitié au moins de leur chiffre d'affaires avec ces commissions, il s'agit plutôt d'une activité marginale pour des entreprises de taille moyenne plutôt plus élevée. Les entreprises concernées par cette activité réalisent vraisemblablement une part de leur chiffre d'affaires en vente d'antiquités ${ }^{3}$.

\section{Les ventes d'objets de récupération} à des particuliers (marbres, meubles...) : 2300 entreprises réalisent un chiffre d'affaires de 104 millions d'euros

Cette activité évoque plutôt la brocante, à la différence de la précédente qui correspond pour partie à l'activité d'antiquaires. Elle concerne deux types d'entreprises. Là encore, deux sous-populations de taille différente - environ 600 entreprises contre un millier - sont relativement spécialisées dans le commerce d'art ( $80 \%$ en moyenne), mais

3. Le cumul d'activités est un aspect qui n'a pu être abordé ici. 
totalisent des chiffres d'affaires de niveau sensiblement différents ${ }^{4}$.

\section{Entreprises relevant de la vente d'occasion en magasin (52.5Z)}

1073 entreprises pèsent 66 millions d'euros dans cette activité. Leur chiffre d'affaires moyen dans l'activité est de $62000 €$, soit $47 \%$ de leur chiffre d'affaires total, ce qui atteste d'une spécialisation limitée. Seules $3 \%$ de ces entreprises emploient plus de 5 salariés.

Parmi ces entreprises, 591 (soit $55 \%$ d'entre elles) réalisent la moitié de leur chiffre d'affaires avec ces biens. Ceci correspond à un chiffre d'affaires moyen dans l'activité de $91000 €$, soit $81 \%$ de leur chiffre d'affaires total. Moins de $1 \%$ de ces entreprises emploient plus de 5 salariés.

482 entreprises réalisent moins de $50 \%$ de leur chiffre d'affaires dans cette activité, avec un chiffre d'affaires moyen de $26000 €$ sur $139000 €$ (19\%). Leur contribution au chiffre d'affaires total de la branche est de 12 millions d'euros.

\section{Entreprises relevant de la vente d'occasion hors magasin (52.6E)}

1319 entreprises réalisent seulement 38 millions d'euros de chiffre d'affaires avec ces biens. Le chiffre d'affaires moyen dans l'activité est de $29000 €(81 \%$ de leur chiffre d'affaires total). Plus de 1000 entreprises sont complètement spécialisées avec un chiffre d'affaires moyen de $29000 €$. Les autres entreprises, réalisant moins de la moitié de leur activité avec ce type de vente, sont au nombre de 280 et réalisent un chiffre d'affaires moyen de $5000 €($ sur $36000 €$, soit $14 \%$ ).

\section{La vente de livres anciens et livres d'occasion : 1332 entreprises réalisent un chiffre d'affaires de 70 millions d'euros ${ }^{5}$}

Ces entreprises sont moins spécialisées cependant : leur chiffre d'affaires moyen est de $53000 €$, ce qui représente une part minoritaire $(31 \%)$ de leur chiffre d'affaires total.

802 entreprises réalisent la moitié au moins de leur chiffre d'affaires, soit $76000 €$ en moyenne (95\% d'un chiffre d'affaires total moyen de $80000 €)$. Elles sont donc fortement spécialisées.
Seules $0,6 \%$ de ces entreprises emploient plus de 5 salariés.

En revanche, les 530 entreprises qui tirent moins de la moitié de leur chiffre d'affaires de la vente de livres anciens ou d'occasion réalisent dans cette activité en moyenne $17000 €$, soit $12 \%$ seulement d'un chiffre d'affaires total beaucoup plus élevé (305000€ en moyenne). Leur contribution au chiffre d'affaires de la branche est faible : 9 millions d'euros. Il s'agit vraisemblablement de l'activité très secondaire de vendeurs importants d'antiquités.

\section{La vente de pièces de collection}

et de timbres-poste de collection :

près de 1000 entreprises réalisent

un chiffre d'affaires de $\mathbf{5 4}$ millions d'euros

Ces deux activités présentent des traits communs quant au nombre d'entreprises concernées (environ 500 ) et à leur caractère de vente très secondaire, audelà d'un petit noyau de spécialistes. Il s'agit donc là de deux domaines de moindre spécialisation que les commerces précédents. Au-delà d'un nombre limité de spécialistes, ces activités constituent toutes deux une part très secondaire de l'activité d'autres vendeurs relevant certainement pour une bonne part des catégories précédentes.

\section{La vente au détail de pièces de collection}

Elle concerne 450 entreprises pour un chiffre d'affaires modique de 30 millions d'euros (69000€ en moyenne sur $158000 €$, soit $43 \%$ ).

Plus de la moitié des entreprises (266) réalisent au moins $50 \%$ de leur chiffre d'affaires avec ces biens, soit $109000 €$ en moyenne sur un total de $139000 €$, soit $78 \%$. Les autres, au nombre de 214, qui en tirent moins de la moitié de leur chiffre d'affaires réalisent en moyenne $19000 €$ sur un total de $182000 €$, soit $10 \%$.

550 entreprises vendent des timbres-poste de collection pour 24 millions d'euros soit, en moyenne, $44000 €$ sur $122000 €$ de chiffre d'affaires total $(36 \%)$.

212 entreprises en tirent plus de la moitié de leur chiffre d'affaires et, en moyenne, $90000 €$ sur $113000 €$, soit $79 \%$. Aucune d'entre elles ne dépasse les 5 salariés. Les autres, au nombre de 338 , réalisent moins de la moitié de leur chiffre d'affaires avec la vente de timbres-poste de collection : $15000 € \operatorname{sur} 127000 €$, soit $12 \%$.

4. Seules une vingtaine d'entreprises réalisent des commissions sur les ventes au détail de ces types de biens pour un chiffre d'affaires marginal (2 millions d'euros).

5. Il faut souligner que la vente de livres anciens et d'occasion est loin d'être intégralement retracée ici, dans la mesure où l'activité de librairie de détail (52.4R) n'est pas prise en compte, alors que la vente accessoire de livres anciens ou d'occasion peut représenter une part importante de l'activité de certaines librairies de livres neufs. 
Tableau 3 - Données de synthèse sur les principales activités et les entreprises concernées

\begin{tabular}{|c|c|c|c|c|}
\hline & $\begin{array}{l}\text { Jommerce d'œuvres } \\
\text { d'art contemporain }\end{array}$ & $\begin{array}{c}\text { Vente } \\
\text { d'antiquités }\end{array}$ & $\begin{array}{l}\text { Vente d'objets } \\
\text { de récupération }\end{array}$ & $\begin{array}{c}\text { Vente de livres } \\
\text { anciens et d'occasion }\end{array}$ \\
\hline Nombre total d'entreprises de la branche & 1491 & 10394 & 2392 & $1332^{\star \star}$ \\
\hline Nombre total d'entreprises du secteur (> $50 \%$ ) & 1171 & 9425 & 1630 & 802 \\
\hline \multicolumn{5}{|l|}{ Nombre total d'entreprises } \\
\hline de la branche hors secteur (<50 \%) & 320 & 971 & 762 & $530^{\star *}$ \\
\hline Chiffre d'affaires de la branche (millions d'euros) & 385 & 1298 & 104 & $70^{\star \star}$ \\
\hline \multicolumn{5}{|l|}{ Chiffre d'affaires total } \\
\hline des entreprises de la branche & 474 & 1425 & 188 & $227^{\star \star}$ \\
\hline Chiffre d'affaires de la branche intra-secteur & $363^{*}$ & 1280 & 92 & 61 \\
\hline Chiffre d'affaires total des entreprises du secteur & $368^{\star}$ & 1343 & 121 & 64 \\
\hline $\begin{array}{l}\text { Estimation des effectifs totaux } \\
\text { des entreprises de la branche }\end{array}$ & 2500 & 6300 & 1000 & 1900 \\
\hline \multicolumn{5}{|l|}{ Estimation des effectifs totaux } \\
\hline des entreprises du secteur & $1900^{\star}$ & 5550 & 550 & 900 \\
\hline
\end{tabular}

Source : DEPS, Ministère de la Culture et de la Communication, 2009

\section{Méthodologie}

Les postes de la nomenclature Naf au niveau détaillé dans lesquels figurent les activités du marché de l'art ont d'abord été identifiés, sans qu'elles soient pour autant individualisées en leur sein. II s'agit des trois postes suivants: " commerce de détail divers en magasin spécialisé » (52.4Z), « commerce de détail de biens d'occasion » (52.4Z) ainsi que "commerce de détail non alimentaire sur éventaire et marchés » (52.6E).

Pour ces trois postes, les données relatives à la ventilation du chiffre d'affaires des entreprises ont été exploitées. La ventilation du chiffre d'affaires est spécifique pour chaque poste et fait apparaître, pour chacun d'eux, des biens et services concernant le marché de l'art. Il est dès lors possible de comptabiliser d'une part les entreprises et leurs effectifs totaux et d'autre part les chiffres d'affaires concernés. Cette exploitation spécifique a porté sur les données relatives à l'année 2006.
La qualité et la pertinence des résultats sont directement liées aux critères d'exploitation retenus et impliquent que les deux conditions suivantes soient réunies:

- les entreprises ayant tout ou partie de leur activité dans le marché de l'art doivent effectivement être concentrées, en termes de code APE, dans les trois postes de la nomenclature retenus. II semble que ce soit le cas, à l'exception de la vente de livres anciens ou d'occasion, que l'on retrouve de manière non négligeable associée à une activité principale de vente de livres neufs;

- la ventilation du chiffre d'affaires doit être correctement effectuée par les entreprises commerciales du marché de l'art dans l'enquête annuelle Entreprises, ce qui reste difficile à déterminer puisque, précisément, seules les entreprises qui procèdent à la ventilation de leur chiffre d'affaires peuvent être identifiées comme telles. On observe que les entreprises de ces trois postes, dans leur ensemble, répondent correctement à cette question comme l'indique le tableau suivant; on peut donc penser qu'il en va de même des entreprises du marché de l'art.

\section{Pratique par les entreprises de la ventilation du chiffre d'affaires réalisé}

\begin{tabular}{|cccc|}
\hline Code APE & $\begin{array}{c}\text { Part des entreprises } \\
\text { ayant effectué une ventilation } \\
\text { de leur chiffre d'affaires } \\
\text { par produit }\end{array}$ & $\begin{array}{c}\text { Part des effectifs des entreprises } \\
\text { ayant effectué une ventilation } \\
\text { de leur chiffre d'affaires } \\
\text { par produit }\end{array}$ & $\begin{array}{c}\text { Part du chiffre d'affaires } \\
\text { des entreprises } \\
\text { ayant effectué une ventilation } \\
\text { de leur chiffre d'affaires } \\
\text { par produit }\end{array}$ \\
$52.4 Z$ & 83 & 88 & 92 \\
$52.5 Z$ & 86 & 97 & 87 \\
$52.6 \mathrm{E}$ & 90 & 72 & 81 \\
\hline
\end{tabular}


ANNEXE 1 - Liste des postes intéressant le marché de l'art pour la ventilation du chiffre d'affaires des entreprises commerciales des secteurs" $52.4 \mathrm{Z}, 52.5 \mathrm{Z}$ et $52.6 \mathrm{E}$

Secteur 52.4Z :

commerce de détail en magasin spécialisé

- Timbres-poste de collection (52.4Z)

- Pièces de collection (52.4Z)

- Euvres d'art contemporain (52.4Z)

Secteur 52.5Z :

commerce de détail de biens d'occasion

- Antiquités, objets d'art et meubles anciens (52.5Z)

- Objets de récupération vendus à des particuliers

(marbres, meubles...) (52.5Z)

- Livres anciens et livres d'occasion (52.5Z)
- Euvres d'art contemporain (52.4Z)

- Commissions sur ventes au détail d'antiquités, objets d'art et meubles anciens (52.5Z)

- Commissions sur ventes au détail d'objets de récupération (marbres, meubles...) (52.5Z)

- Activités juridiques (expertise d'œuvres d'art) (74.1A)

Secteur 52.6E :

commerce de détail hors magasin

- Antiquités, objets d'art et meubles anciens (52.5Z)

- Objets de récupération vendus à des particuliers

(marbres, meubles...) (52.5Z)

* Est indiqué entre parenthèses le poste de la nomenclature auquel est rattachée l'activité correspondante.

ANNEXE 2 - Données détaillées

\begin{tabular}{|c|c|c|c|c|c|c|c|c|c|c|}
\hline & $\begin{array}{l}\text { CEuvres } \\
\text { d'art } \\
\text { contem- } \\
\text { porain }\end{array}$ & $\begin{array}{c}\text { CEuvres } \\
\text { d'art } \\
\text { contem- } \\
\text { porain }\end{array}$ & $\begin{array}{c}\text { Antiquités, } \\
\text { objets } \\
\text { d'art, } \\
\text { meubles } \\
\text { anciens }\end{array}$ & $\begin{array}{c}\text { Antiquités, } \\
\text { objets } \\
\text { d'art, } \\
\text { meubles } \\
\text { anciens }\end{array}$ & $\begin{array}{l}\text { Objets } \\
\text { de récup. } \\
\text { vendus } \\
\text { à des } \\
\text { particuliers }\end{array}$ & $\begin{array}{c}\text { Objets } \\
\text { de récup. } \\
\text { vendus } \\
\text { à des } \\
\text { particuliers }\end{array}$ & $\begin{array}{c}\text { Livres } \\
\text { anciens } \\
\text { et } \\
\text { d'occasion }\end{array}$ & $\begin{array}{l}\text { Pièces } \\
\text { de } \\
\text { collection }\end{array}$ & $\begin{array}{c}\text { Timbres } \\
\text { de } \\
\text { collection }\end{array}$ & $\begin{array}{c}\text { Activités } \\
\text { juridiques } \\
\text { (expertise) }\end{array}$ \\
\hline & $52.4 Z$ & $52.5 Z$ & $52.5 Z$ & $52.6 \mathrm{E}$ & $52.5 Z$ & $52.6 \mathrm{E}$ & $52.5 Z$ & $52.5 Z$ & $52.5 Z$ & $52.5 Z$ \\
\hline Nombre d'entreprises & 1356 & 135 & 6203 & 4191 & 1073 & 1319 & 1332 & 40 & 550 & 168 \\
\hline Chiffre d'affaires activité ${ }^{*}$ & 380 & 5 & 1135 & 163 & 66 & 38 & 70 & 33 & 24 & 3 \\
\hline Chiffre d'affaires total* & 441 & 33 & 1255 & 170 & 141 & 47 & 227 & 76 & 67 & 56 \\
\hline \multicolumn{11}{|l|}{ Chiffre d'affaires } \\
\hline Chiffre d'affaires moyen total ${ }^{* *}$ & 325 & 244 & 202 & 41 & 131 & 36 & 170 & 158 & $\begin{array}{r}44 \\
122\end{array}$ & 333 \\
\hline$\%$ de l'activité totale & 86 & 15 & 90 & 96 & 47 & 81 & 31 & 43 & 36 & 5 \\
\hline \multicolumn{11}{|c|}{ dont plus de $50 \%$ du chiffre d'affaires dans l'activité } \\
\hline Nombre d'entreprises & 1170 & $\mathrm{C}$ & 5696 & 3729 & 591 & 1039 & 802 & 266 & 212 & 0 \\
\hline Chiffre d'affaires activité* & 363 & C & 1118 & 162 & 54 & 37 & 61 & 29 & 19 & 0 \\
\hline Chiffre d'affaires total* & 368 & C & 1178 & 165 & 74 & 37 & 64 & 37 & 24 & 0 \\
\hline \multicolumn{11}{|l|}{ Chiffre d'affaires } \\
\hline moyen activité ${ }^{\star *}$ & 310 & C & 196 & 43 & 91 & 29 & 76 & 109 & 90 & 0 \\
\hline Chiffre d'affaires moyen total ${ }^{\star *}$ & 315 & C & 207 & 44 & 125 & 29 & 80 & 139 & 113 & 0 \\
\hline$\%$ de l'activité totale & 99 & C & 95 & 98 & 73 & 100 & 95 & 78 & 79 & III \\
\hline \multicolumn{11}{|c|}{ dont moins de $50 \%$ du chiffre d'affaires dans l'activité } \\
\hline Nombre d'entreprises & 182 & C & 507 & 462 & 482 & 280 & 530 & 214 & 338 & 168 \\
\hline Chiffre d'affaires activité ${ }^{*}$ & 17 & C & 17 & 1 & 12 & 1 & 9 & 4 & 5 & 3 \\
\hline Chiffre d'affaires total* & 73 & C & 77 & 5 & 67 & 10 & 163 & 39 & 43 & 56 \\
\hline \multicolumn{11}{|l|}{ Chiffre d'affaires } \\
\hline moyen activitét* & 93 & C & 35 & 3 & 26 & 5 & 17 & 19 & 15 & 18 \\
\hline Chiffre d'affaires moyen total ${ }^{\star *}$ & 398 & C & 152 & 11 & 139 & 36 & 305 & 182 & 127 & 333 \\
\hline$\%$ de l'activité totale & 23 & C & 23 & 20 & 19 & 14 & 12 & 10 & 12 & 5 \\
\hline
\end{tabular}

Source : DEPS, Ministère de la Culture et de la Communication, 2009

\section{L'essentiel en quelques chiffres}

13505 entreprises spécialisées dans le commerce de l'art • 1914 millions d'euros de chiffre d'affaires réalisé par ces entreprises spécialisées • 9000 emplois dans ces entreprises spécialisées • Taux de spécialisation de $95 \%$ dans l'activité 